\title{
A study for the static properties of symmetric linear multiblock copolymers under poor solvent conditions
}

\author{
Panagiotis E. Theodorakis \\ Faculty of Physics, University of Vienna, Botlzmanngasse 5, A-1090 Vienna, Austria \\ Institute for Theoretical Physics and Center for Computational Materials Science, \\ Vienna University of Technology, Hauptstraße 8-10, A-1040 Vienna, Austria and \\ Vienna Computational Materials Laboratory, Sensengasse 8/12, A-1090 Vienna, Austria \\ Nikolaos G. Fytas \\ Departamento de Física Teórica I, Universidad Complutense, E-28040 Madrid, Spain
}

(Dated: October 31, 2018)

\begin{abstract}
We use a standard bead-spring model and molecular dynamics simulations to study the static properties of symmetric linear multiblock copolymer chains and their blocks under poor solvent conditions in a dilute solution from the regime close to theta conditions, where the chains adopt a coil-like formation, to the poorer solvent regime where the chains collapse obtaining a globular formation and phase separation between the blocks occurs. We choose interaction parameters as is done for a standard model, i.e., the Lennard-Jones fluid and we consider symmetric chains, i.e., the multiblock copolymer consists of an even number $n$ of alternating chemically different $\mathrm{A}$ and $\mathrm{B}$ blocks of the same length $N_{A}=N_{B}=N$. We show how usual static properties of the individual blocks and the whole multiblock chain can reflect the phase behavior of such macromolecules. Also, how parameters, such as the number of blocks $n$ can affect properties of the individual blocks, when chains are in a poor solvent for a certain range of $n$. A detailed discussion of the static properties of these symmetric multiblock copolymers is also given. Our results in combination with recent simulation results on the behavior of multiblock copolymer chains provide a complete picture for the behavior of these macromolecules under poor solvent conditions, at least for this most symmetrical case. Due to the standard choice of our parameters, our system can be used as a benchmark for related models, which aim at capturing the basic aspects of the behavior of various biological systems.
\end{abstract}

PACS numbers: 87.15.A, 87.15.N, 87.15.B, 82.35.Pq, 82.35.Jk

\section{INTRODUCTION}

Polymers combining chemically different monomeric units on their structure found very much theoretical and experimental interest, as, for instance, diblock copolymers, where in the case of a linear chain one block of the chain consists of one type of monomers, while the other part of another type of monomers. These macromolecules have already found particular interest as they form different structures depending on their structural parameters, thermodynamics conditions, etc. For a standard theoretical review one could see Ref. [1] and references therein. We underline that the motivation for studying such systems stems from the fact that these materials are used in industrial scale [1] and also in very advanced technological applications (as an example one may look a very recent review Ref. [2]). As an extension, regarding possible complexity in the structure or other aspects, of diblock copolymers is regarded multiblock copolymers, where in their simplest case more than two blocks comprise a linear polymeric chain and the blocks are composed either of type A or type B monomers. The phase diagram of multiblock copolymer melts [3] resembles the phase diagram of diblock copolymer chains [4 6 ], where the scaling parameter $\chi N$ controls the phase behavior of the system, as it has also been discussed in simulation studies [7]. For the most symmetrical case the lamel- lar structure is predicted, an approach that is particular valid in the strong segregation limit. The case of multiblock copolymer chains is particularly interesting even when only two type of blocks (A, B) alternating along a linear chain in an infinite dilute solution is considered 8 -15]. In this case, it is sufficient to study the behavior of an isolated chain taking into account only interactions (often of short range) within the chain. A model describing a multiblock copolymer chain could also exhibit some interest due to its close relation to various toy-models [16], which try to mimic the behavior of various biomacromolecules in several processes [17. Such biomacromolecules are rather formed by periodically repeated structural units ("monomers") along their chain. Simple (coarse-grained) models [18] incorporating the key aspects for a specific biomacromolecule are able to capture efficiently their relevant properties for which the model is designed.

The phase behavior of a single multiblock copolymer chain has been extensively studied by means of molecular dynamics (MD) simulations by using a standard bead spring-model and considering interactions between beads according to a standard model (Lennard-Jones (LJ) fluid) [13 15]. Of course the use of MD simulations imposes certain limits in the range that the parameters may vary, for example, the range of the temperature and the chain length. However, it has been shown that the 
different regimes of interest for the phase behavior can all be accessed by simulations $8-10,13$ 15, 18]. Multiblock copolymers composed of two different types of blocks (A and B) alternating along the linear chain and length of a block A being equal to the length of block B that adopt coil-like structures in a good solvent or at temperatures close to the $\Theta[11$ 15, 19] have been studied. The chemical difference of monomers should actually be kept responsible for an expansion in the chain dimensions with respect to the equivalent homopolymer chains (same total number of monomers) under the same thermodynamic conditions [11, 12]. Below a certain temperature [13] a multiblock copolymer chain collapses due to the incompatibility between monomers and the solvent obtaining the so-called globule formation and different scenarios of phase behavior have been discussed [14, 15]. Simulations have confirmed the validity of the parameter $\chi N$ in defining the phase behavior of a di/multi-block copolymer system, but it has also been discussed that the geometry of the microphase separated regions is controlled by the total number of blocks $n$, as well as other parameters, i.e. the relative size and arrangement of the blocks [7]. The same has been shown to be true for a single multiblock copolymer chain under poor solvent conditions [13 15].

The phase behavior of such macromolecules has been discussed within the frame of the analysis of the formed clusters (which contain two or more blocks of the same type) and of the probability that a cluster of one type of blocks contains all A blocks while another cluster contains all B blocks [13 15], i.e. a single lamellar structure with an A-B interface between blocks of type A and blocks of type B. Thus, when this probability is unity we fall into the above single lamellar case. Then, for the range of temperatures $(\chi \propto \Delta \varepsilon / T, \Delta \varepsilon=$ const $)$ and of chain lengths $(n N$, where $N$ is the block length; blocks A and $B$ have the same length) accessible to simulations this type of phase behavior has been seen for small number of blocks $n$ and for values of block length $N>20$. Another scenario suggests that the above type of phase behavior takes place with a certain probability $\left(P_{N_{c l}=2}<1, N_{c l}\right.$ being the number of clusters; such probability can be very high or very small depending on the values of $N, n$, and $T)$. The third scenario corresponds to the case that the probability $P_{N_{c l}=2}=0$ (the formation of only two clusters with monomers of different type never occurs) and a symmetric variation in the number of clusters $N_{c l}$ around an average value $\left(2<N_{c l}<n, n\right.$ is the total number of blocks of the chain) is seen. Of course, the analysis of the probability is performed separately for clusters of type A monomers and for clusters of type B monomers. Otherwise the A and B beads that belong to the same chain would always belong to a single cluster containing all the beads of the chain. Similar conclusions have been also drawn by a similar model [8 10], where also the geometry of these cluster phases has been analyzed in detail. Guided by the theory [3], one would rather expect in the long chain limit that a ground-state-type structure would be a single lamellar domain, where an in-

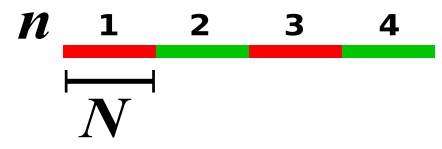

FIG. 1: (Color online) Definition of structural parameters describing our linear multiblock copolymer chains. $n$ (in this case $n=4$ ) is the number of different blocks $\mathrm{A}$ and $\mathrm{B}\left(n_{A}=n_{B}=n / 2\right)$ denoted with different color (or grey tone) and $N$ is the length of each block. All the blocks, irrespective of whether they are of type A or B, have the same length $N$. Then the total length of the chain is $n N$.

terface between all A- and B-type blocks is formed, similarly to what is known for multiblock copolymer melts for symmetric choice of parameters. Such a structure would have much less (unfavorable) A-B contacts compared to a multidomain structure of $\mathrm{A}$ and $\mathrm{B}$ clusters, which is kinetically favored in simulations. Recently, the static properties of the formed clusters for such systems have been discussed [13], where one is also able to detect in addition to the phase boundaries the globule-coil crossover and the proximity of the $\Theta$ temperature through such an analysis. In this manuscript, we will show further, that properties such as the bond correlation and asphericity can provide a rather accurate evidence for detection of full phase separation (single lamellar structure) in multiblock copolymer chains. Additionally, we will argue that these properties can provide information on the length $N$ and the number of blocks $n$ that this phase behavior is taking place.

Furthermore, we provide a complete understanding and a broad discussion of the static properties of such macromolecules under poor solvent conditions for the blocks and the multiblock chain as a whole, at least for the most symmetric case that is considered here, and for chain lengths and thermodynamic conditions accessible to our simulations. As we have chosen in our study the same potential parameters as for the standard LJ fluid [20], our results could also serve as a benchmark for similar polymeric and biological systems. We discuss our results only in the poor solvent regime, which is the most interesting case due to the different phase behavior that has been observed when the chain is in the collapsed state (globule state) [13 15].

The rest of the paper is organized as follows. In Sec. II we describe our simulation model and the methods to analyze our results. In Sec. III we present our results for the whole multiblock chain and the individual blocks and we discuss particular details of the studied properties pertinent to the phase behavior of the multiblock chains. Finally, in Sec. IV] we summarize our conclusions in connection with recent results on the phase behavior of symmetric linear multiblock copolymers. 


\section{MODEL AND METHODS TO ANALYZE THE SIMULATIONS}

As a detailed study of the systems under consideration is computationally demanding for reasons that will be discussed below, we have focused only on symmetric linear multiblock copolymers, where blocks of type A beads and blocks of type B beads alternate along the chain. Then the length of all blocks, irrespective of whether being of type $\mathrm{A}$ or $\mathrm{B}$, is $N$, the number of A-type blocks $n_{A}$ is equal to the number of B-type blocks $n_{B}$, with $n=n_{A}+n_{B}$ an even number denoting the total number of blocks. A multiblock copolymer chain is described by the parameters shown schematically in Fig. 1] where the different colors correspond to the different types of blocks, which alternate along the chain.

To model our chains we use the standard bead-spring model [21 26] to describe a multiblock copolymer chain, where all beads interact with a truncated and shifted LJ potential $U_{L J}(r)$ and nearest neighbors bonded together along the chain also experience the finitely extensible nonlinear elastic potential $U_{F E N E}(r), r$ being the distance between the beads. Thus,

$$
U_{L J}^{\alpha \beta}(r)=4 \varepsilon_{L J}^{\alpha \beta}\left[\left(\sigma_{L J}^{\alpha \beta} / r\right)^{12}-\left(\sigma_{L J}^{\alpha \beta} / r\right)^{6}\right]+C, \quad r \leq r_{c}
$$

where $\alpha, \beta=A, B$ denote the different type of monomers, and the constant $C$ is defined such that the potential is continuous at the cut-off $\left(r_{c}=2.5\right)$. Henceforth units are chosen such that $\varepsilon^{\alpha \alpha}=1, \sigma^{\alpha \alpha}=1$, the Boltzmann constant $k_{B}=1$, and in addition the mass $m_{L J}^{\alpha \beta}$ of beads is chosen to be unity. For simplicity, $\sigma_{L J}^{\alpha \beta}=1$, but $\varepsilon_{L J}^{A A}=\varepsilon_{L J}^{B B}=2 \varepsilon_{L J}^{A B}=1$, in order to create an unmixing tendency between monomers $\mathrm{A}$ and $\mathrm{B}$ belonging to different blocks as done in previous studies [13 15, 2729], and as is used for a standard system (LJ fluid) [20]. Therefore, $\Delta \varepsilon=\varepsilon_{L J}^{A B}-1 / 2\left(\varepsilon_{L J}^{A A}+\varepsilon_{L J}^{B B}\right)$ was kept the same throughout our simulations and $\chi(\chi \propto \Delta \varepsilon / T)$ was mainly varied by tuning the temperature $T$. The potential of Eq. (1) acts between any pair of beads, irrespective of whether they are bonded or not. For bonded beads additionally the potential $U_{F E N E}(r)$ acts,

$$
U_{\mathrm{FENE}}=-\frac{1}{2} k r_{0}^{2} \ln \left[1-\left(r / r_{0}\right)^{2}\right], \quad 0<r \leq r_{0} .
$$

where $U_{F E N E}\left(r \geq r_{0}\right)=\infty$, and hence $r_{0}$ is the maximal distance between bonded beads. We use the standard choice $r_{0}=1.5$ and $k=30$. We recall that for linear chains the $\Theta$ temperature for the present model has been roughly estimated as $T_{\Theta} \approx 3.0$ (a more accurate value [30] $T_{\Theta} \approx 3.18$ could only be established for chain length $n N>200, n=1)$. Thus, we have considered in our study the range of temperatures $1.5 \leq T \leq 3.0$. Moreover, in previous discussion of this system [13] we showed that the chain enters in the globule regime at temperature $T \approx 2.4$. Therefore, phase separation between $\mathrm{A}$ and $\mathrm{B}$ monomers takes place below this temperature.
For homopolymer chains this boundary is sharper compared to the case of multiblock copolymers where also appears smoother as the number of blocks $n$ increases for constant chain length $n N$ as the A-B junctions between blocks increase. Of course, the temperature that we enter the globule regime (upon lowering the temperature) is the same for homopolymer and copolymer chains, because in our model the location of this boundary depends only on the solvent (controlled by temperature in our simulations), which is the same for a homopolymer chain, as well as for type A and type B beads of a copolymer chain, but in the case of copolymer chains it only appears smoother.

Further on our simulation method, the positions $\vec{r}_{i}(t)$ of the effective monomers with label $i$ evolve in time $t$ according to Newton's equation of motion, amended by the Langevin thermostat

$$
m \frac{d^{2} \vec{r}_{i}}{d t^{2}}=-\nabla U_{i}-\gamma \frac{d \vec{r}_{i}}{d t}+\vec{\Gamma}_{i}(t)
$$

where $U_{i}\left(\vec{r}_{j}(t)\right)$ is the total potential acting on the $i$ 'th bead due to its interactions with the other beads at sites $\vec{r}_{j}(t), \gamma$ is the friction coefficient, and $\vec{\Gamma}_{i}(t)$ the associated random force. The latter is related to $\gamma$ by the standard fluctuation-dissipation relation

$$
\left\langle\vec{\Gamma}_{i}(t) \cdot \vec{\Gamma}_{j}\left(t^{\prime}\right)\right\rangle=6 k_{B} T \gamma \delta_{i j} \delta\left(t-t^{\prime}\right) .
$$

Following previous work [21 30], we choose $\gamma=0.5$, the MD time unit $\tau_{L J}=\left(m \sigma_{L J}^{2} / \varepsilon_{L J}^{\alpha \alpha}\right)^{1 / 2}=1$ being also unity for our choice of units. Equation (3) is integrated using the leap-frog algorithm [31], with a time step $\Delta t=$ $0.006 \tau_{L J}$ and utilizing the GROMACS package [32].

Simulation of polymer chains under poor solvent conditions is notoriously difficult as relaxation times become exceedingly high. Therefore, we describe here briefly our simulation procedure. After equilibration at a "high" temperature (in the coil regime), we collect a number of independent samples (typically 500), which we use as initial configurations for slow cooling runs, as is done in previous studies [27 29]. For longer chains, temperatures higher than $T=3.0$ were used in order to facilitate the procedure of obtaining initial independent samples. We note here that the solvent is taken into account in our model only implicitly by tuning the temperature [1315]. Then, decrease of the temperature corresponds to higher incompatibility of the implicit solvent with the monomers. For each cooling history, we lower the temperature in steps of $\Delta T=0.1$ and run the simulations for $2 \times 10^{6} \mathrm{MD}$ steps using the final configuration at each (higher) temperature as starting configuration for the next (lower) temperature. At low enough temperatures [14] (typically $T<2.0$, at a rather high distance from the $\Theta$ temperature), where dense "clusters" of a few neighboring blocks are formed, it is not possible to run simple MD simulations long enough to sample the phase space adequately. Therefore, using this procedure of independent cooling histories is indispensable for obtaining meaningful statistics. This large statistical effort 
prevents the study of exceedingly long chains for this simulation model.

At temperatures close to $\Theta$, coil-like structures are formed and the differences between multiblock copolymer chains with different $n, N$ are rather small, expecting that at $T \rightarrow \infty$ these differences will completely disappear. At temperatures below $T \approx 2.4$ the chains collapse and blocks of the same type of monomers form clusters with other monomers belonging to different blocks, due to the unfavorable interactions between $\mathrm{A}$ and $\mathrm{B}$ beads $13-$ 15]. The static properties of these clusters have been described in detail in previous communication [13]. Here, we focus on the static properties of the whole chain (of length $n N$ ) as well as the static properties of individual blocks of $N$ effective monomers. Due to the symmetric choice of our parameters, the averages of the static properties for A and B blocks should not differ. Therefore, the calculation for averages of the properties of the blocks should be taken over all blocks $n$, irrespective of their type, and their properties will be denoted with the subscript "b", for example the average value of the gyration radius of individual blocks would read $R_{g, b}$ while those for the whole chains without this index, namely as $R_{g}$. We discuss below the properties that might need some more details.

We follow the definition of standard textbooks 33 35] to discuss aspects related to the persistence length of the polymer chains, where the persistence length is defined from the decay of bond orientational correlations along the chain. However, one should be careful when defines the persistence length with this correlation function as it has been discussed elsewhere [36, 37]. Nevertheless, we show in this work that this property is extremely accurate in indicating the appearance of full phase separation of the blocks in symmetric multiblock copolymers. We give here a brief definition of this property. Defining the bond vectors $\vec{\alpha}_{i}$ in terms of the monomer positions $\vec{r}_{i}$ as $\vec{\alpha}_{i}=\vec{r}_{i+1}-\vec{r}_{i}, i=1, \ldots, n N-1$, the bond orientational correlation is defined as

$$
\langle\cos \theta(s)\rangle=l_{b}^{-2} \frac{1}{N_{b}-1-s} \sum_{i=1}^{N_{b}-1-s}\left\langle\vec{\alpha}_{i} \cdot \vec{\alpha}_{i+s}\right\rangle .
$$

Note $\vec{\alpha}_{i}^{2}=l_{b}^{2}$, with $l_{b}$ being the average bond length. For this model $l_{b} \approx 1.00$ and hence $\langle\cos \theta(0)\rangle \approx 1$, of course. Considering the limit $n N \rightarrow \infty$ and assuming Gaussian chain statistics, one obtains an exponential decay, since then $\langle\cos \theta(s)\rangle=\langle\cos \theta(1)\rangle^{s}=\exp [\ln \langle\cos \theta(1)\rangle]$, and thus

$$
\langle\cos \theta(s)\rangle=\exp \left[-s / l_{p}\right], l_{p}^{-1}=-\ln \langle\cos \theta(1)\rangle .
$$

For chains at the $\Theta$ point [38] or in melts [39]

$$
\langle\cos \theta(s)\rangle \propto s^{-3 / 2}, s \rightarrow \infty .
$$

Below $\Theta$ temperature this correlation function falls-off rapidly at small $s$ depending strongly on temperature $T$.

In our study we also consider properties related to the shape of multiblock copolymers. We follow the description of Theodorou and Suter [40] to define the apshericity

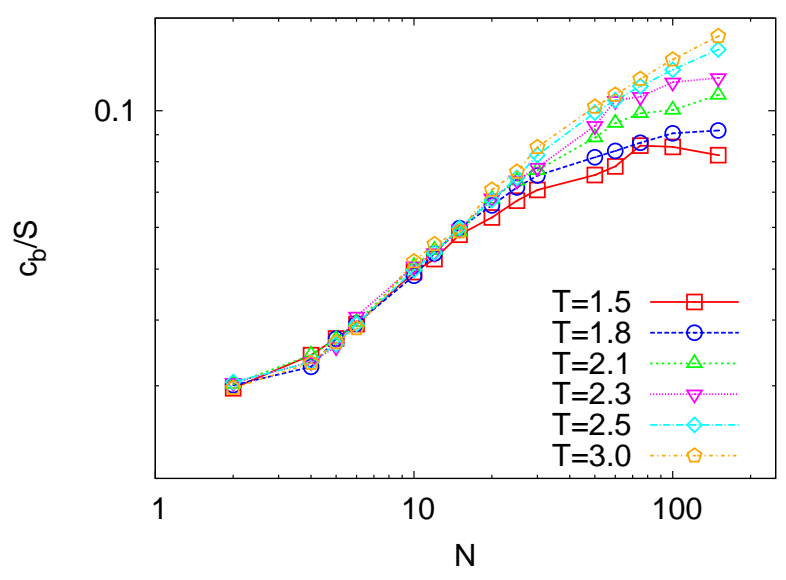

(a)

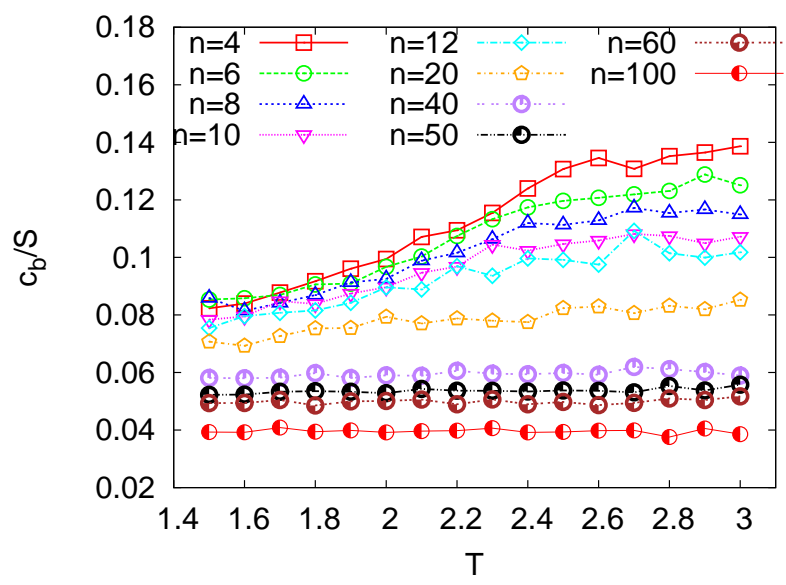

(b)

FIG. 2: (Color online) Acylindricity of the blocks for chains of total lentgh $n N=600$. The dependence with the block length $N$ is shown for different temperatures

(a) and the dependence with the temperature $T$ for different number of blocks $n$ (b).

and acylindricity of a mutliblock copolymer chain. First, we define the gyration tensor as

$$
\mathbf{S}=\frac{1}{N_{\xi}} \sum_{i=1}^{N_{\xi}} \mathbf{s}_{i} \mathbf{s}_{i}^{T}=\overline{\mathbf{s s}^{T}}=\left[\begin{array}{lll}
\overline{x^{2}} & \overline{x y} & \overline{x z} \\
\overline{x y} & \overline{y^{2}} & \overline{y z} \\
\overline{x z} & \overline{y z} & \frac{z^{2}}{2}
\end{array}\right],
$$

where $\mathbf{s}_{i}=\operatorname{col}\left(x_{i}, y_{i}, z_{i}\right)$ is the position vector of each bead, which is considered with respect to the center of mass of the beads $\sum_{i=1}^{N_{\xi}} \mathbf{s}^{i}=0$, and the overbars denote an average over all beads $N_{\xi}$. When the gyration tensor of the whole chain is considered, then $N_{\xi}=n N$. For the blocks $N_{\xi}=N$, the gyration tensor and the properties are calculated for each block separately and then an average over all blocks irrespective of their type is taken. As mentioned earlier, this is perfectly valid due to the 
symmetry of our model. The gyration tensor is symmetric with real eigenvalues and a cartesian system that this tensor is diagonal can always be found, i.e.,

$$
\mathbf{S}=\operatorname{diag}\left(\overline{X^{2}}, \overline{Y^{2}}, \overline{Z^{2}}\right),
$$

where the axes are also chosen in such way that the diagonal elements (eigenvalues of $\mathbf{S}) \overline{X^{2}}, \overline{Y^{2}}$, and $\overline{Z^{2}}$ are in descending order $\left(\overline{X^{2}} \geq \overline{Y^{2}} \geq \overline{Z^{2}}\right)$. These eigenvalues are called the principal moments of the gyration tensor. From the values of the principal moments, one defines quantities such as the asphericity $b$,

$$
b=\overline{X^{2}}-1 / 2\left(\overline{Y^{2}}+\overline{Z^{2}}\right) .
$$

When the particle distribution is spherically symmetric or has a tetrahedral or higher symmetry, then $b=0$. The acylindricity $c$

$$
c=\overline{Y^{2}}-\overline{Z^{2}}
$$

is zero when the particle distribution is in sync with a cylindrical symmetry. Therefore, the acylindricity and asphericity are the relevant quantities that would describe geometries that could be relevant in the case of a homopolymer or multiblock chain. These quantities are taken with respect to $S$, to the sum of the eigenvalues, i.e. the square gyration radius of the chain, which we also have calculated independently on our original cartesian coordinates in order to check our results.

Assuming an ellipsoidal shape and based on the calculation of the above eigenvalues of the gyration tensor, one can define an effective volume expressed by the following relation [41]

$$
V^{e f f}=4 \pi \sqrt{3} \prod_{i=1}^{3} \sqrt{\lambda_{i}},
$$

where $\lambda_{1}=\overline{X^{2}}, \lambda_{2}=\overline{Y^{2}}$, and $\lambda_{3}=\overline{Y^{2}}$, are the eigenvalues of the radius of gyration tensor. Then, the effective radius of a sphere with the same volume as this ellipsoid $\left(V^{e f f}\right)$ is given by the geometrical mean of individual radii $R_{g}^{e f f}=\sqrt{3} \prod_{i=1}^{3} \sqrt[6]{\lambda_{i}}$. This can be compared with the volume of an effective sphere defined by the gyration radius $R_{g}=\sqrt{\sum_{3}^{i=1} \lambda_{i}}$. Such an analysis is not in the scope of the present study.

Another property that provides information on the microscopic properties of multiblock chains is the number of contacts between beads of the same or different type. Smaller number of contacts A-B suggests a tendency towards phase separation. The number of contacts can be mathematically described by the following formula [13, 29]

$$
n_{\alpha \beta}=4 \pi \int_{0}^{r_{n}} g_{\alpha \beta}(\Delta r)(\Delta r)^{2} d(\Delta r),
$$

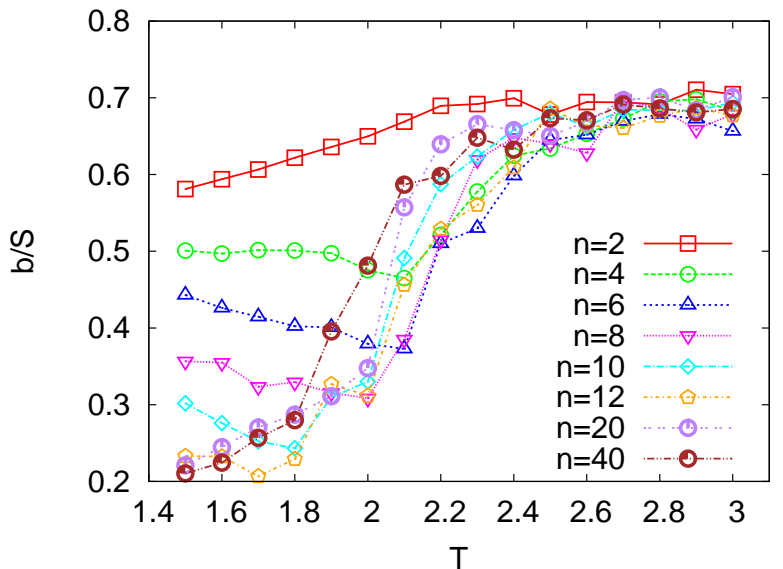

FIG. 3: (Color online) The dependence of asphericity with the temperature $T$ for different combinations of $n$,

$N$ for chains of total length $n N=600$ is shown.

where $\Delta r$ is the absolute value of the distance between two sites of monomers $\vec{r}_{i}, \vec{r}_{j}$ in the multiblock copolymer chain, and $g_{\alpha \beta}$ the corresponding radial distribution function. Equation (13) means that a pair of monomers $[\alpha, \beta,(\alpha, \beta=A, B)]$ is defined to have a pairwise contact if their distance is less than $r_{n}$. For the distance $r_{n}$ we have used the standard Stillinger [42] neighborhood criterion for monomers. We followed the standard choice $r_{n}=1.5 \sigma_{L J}^{\alpha \beta}$ and checked that qualitatively very similar results were obtained if one chooses $r_{n}$ a bit smaller than this choice (larger values of $r_{n}$ are physically hardly significant, since then the particles are too weakly bound, due to the rapid fall-off of the LJ potential). Therefore, a pair of monomers being an absolute distance less than $r_{n}=1.5$ apart define a "contact". Then, the numbers presented in this manuscript will denote the average number of neighbors per monomer.

\section{RESULTS}

Firstly, we discuss properties related to the acylindricity and asphericity of the chains. The acylindricity defines the deviation of the shape of the chain from a cylindrical geometry; correspondingly the asphericity expresses the deviation from a spherical shape or tetrahedral or higher symmetry with respect to the total dimensions of the chains. Then Fig. 2 a presents the dependence of the acylindricity of individual blocks on average for multiblock copolymer chains of the same total length $n N=600$ on the block length $N$. Thus, the number of blocks for each chain is $n=600 / N$. This choice for the total length of the multiblock chain allows for the observation of all interesting regimes related to the phase behavior of such macromolecules [13 15]. In Fig. 2a one 


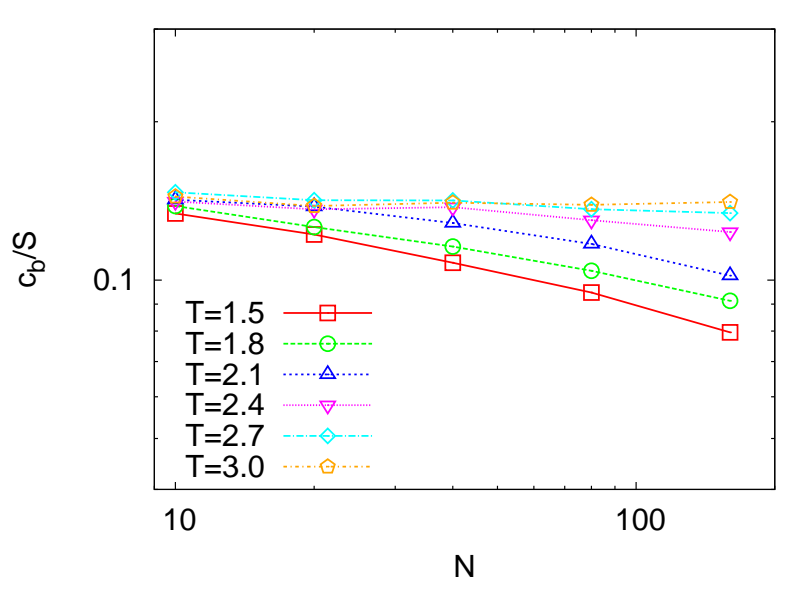

(a)

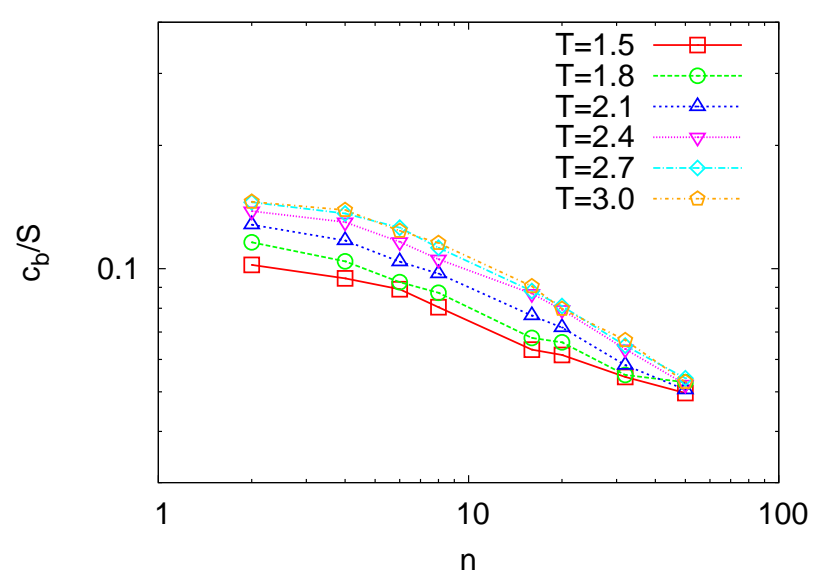

(b)

FIG. 4: (Color online) (a) Acylindricity of the the blocks of a chain with $n=8$ blocks as a function of the block length $N$ for different temperatures, as indicated. The dependence of acylindricity on the number of blocks $n$ is shown correspondingly in part (b) for $N=80$.

can observe very clearly a regime for intermediate values of $2<N<20$ where the curves show small deviations from one another. The cases of $N=2$ and $N=1$ are particular. For $N=1$ (not shown) the acylindricity drops to zero. At lower temperatures larger deviations for higher values of $N$ are seen. Nevertheless from such a plot we are not able to find any hint that relates to the wellknown behavior of these systems 13 15. Looking at the dependence of the above systems with different $N$ on the temperature $T$ (Fig. 2b) it is clearer that small blocks show very small dependence on the temperature due to the very small size of the blocks $(N=600 / n)$, as it also happens in the plot of Fig. $2 \mathrm{a}$. The graph of Fig. $2 \mathrm{~b}$ indicates that for small values of $N$, i.e. $n>20$, the blocks rather do not show any dependence on the temperature,

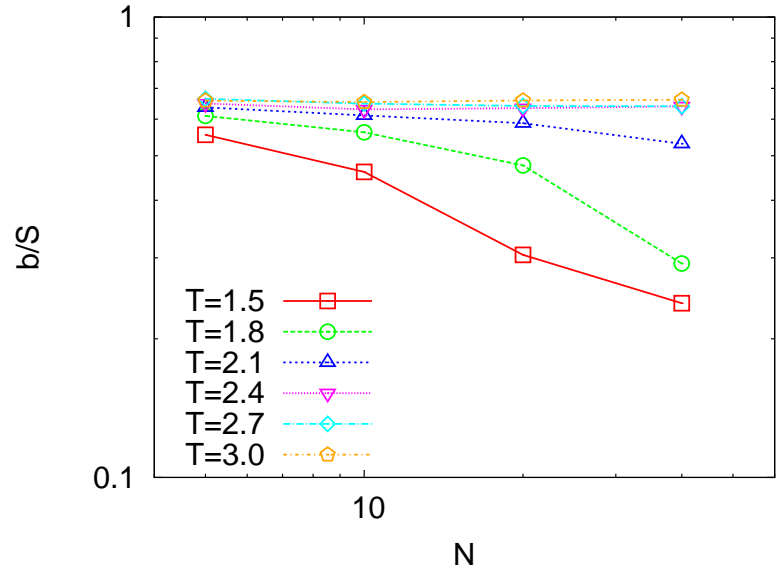

(a)

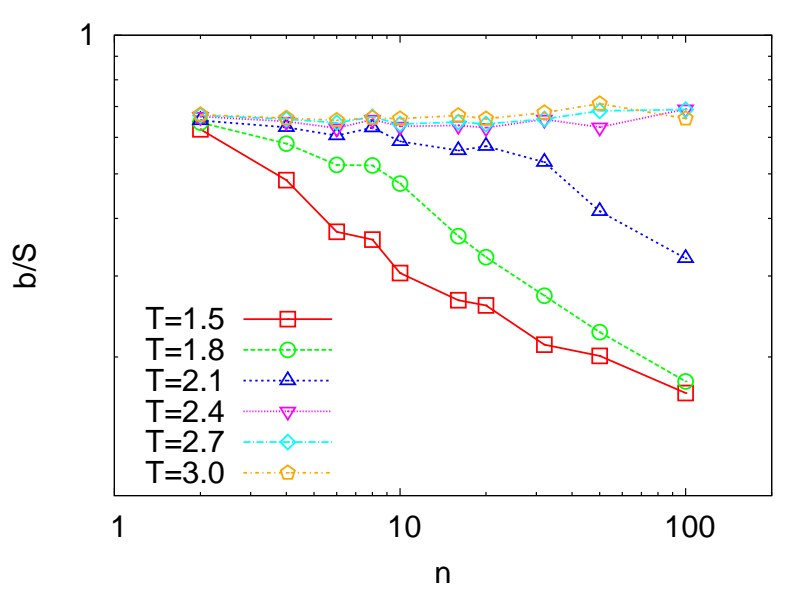

(b)

FIG. 5: (Color online) Asphericity of chains composed of $n=10$ blocks as a function of the block length $N$ (a), and asphericity of chains with block length $N=20$ as a function of the number of blocks $n$ (b).

while for $n<20(N>30)$, stronger dependencies on the the temperature $T$ are seen. This suggests that stronger deviations from a cylidrical symmetry persist for multiblock chains of higher $N$, as $n N$ remains constant. This behavior is also related to the behavior of the size of the cluster formations in these systems, which has been discussed elsewhere 13]. From the results of Fig. 2 we do not see any direct correlation to the phase behavior of the multiblock chains by examining the shape of the individual blocks.

On the other hand, the asphericity of the whole multiblock chain of total length $n N=600$ shows interesting behavior when a plot with the temperature is attempted. We remind the reader that the total chain length $n N=600$ is high enough to consider all the different cases of phase behavior of symmetric multi- 


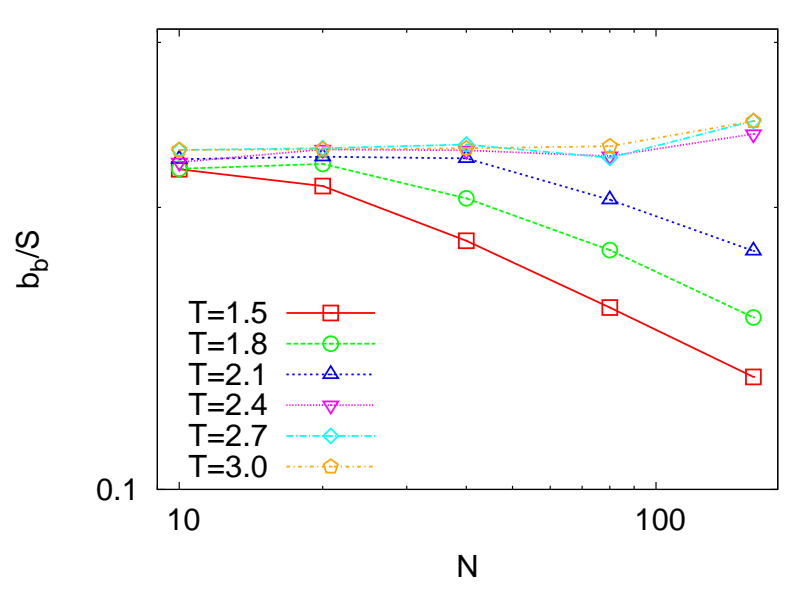

(a)

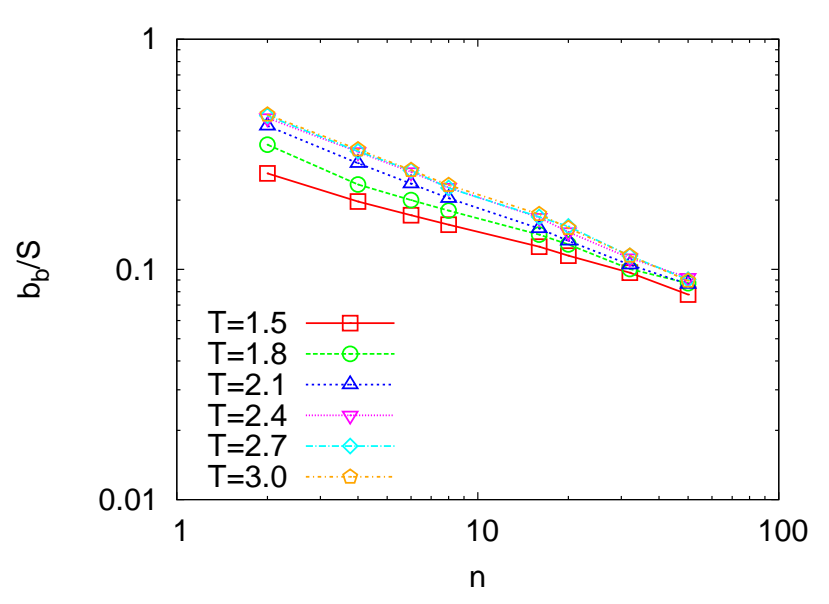

(b)

FIG. 6: (Color online) Asphericity of the blocks of multiblock chains with $n=8$ as a function of the block length $N$ (a), and of chains with block length $N=80$

(b) as a function of the number of blocks $n$.

block copolymers, which have already been discussed in detail [14, 15]. Figure 3 then illustrates two different regimes. At rather high temperatures $(T>2.4)$ the different multibock chains (of the same total length $n N$ ) exhibit small differences as we are close to $\Theta$ conditions. Such small differences are related to the number of proxima A-B interactions at the junctions that connect the consecutive blocks A-B blocks. The values on the $y$-axis of this plot indicate strong deviation of the chain's shape from a spherical one, due to the excluded volume interactions in combinations with the incompatibility between monomers of type $\mathrm{A}$ and $\mathrm{B}$ at the junctions of $\mathrm{A}$ and B blocks. At high values of $N$ (small $n$ except for the case $n=2$ which will be separately discussed below) we observe strong differences at low temperatures. The case $n=4$ reaches at temperature close to $T=2.2 \mathrm{a}$

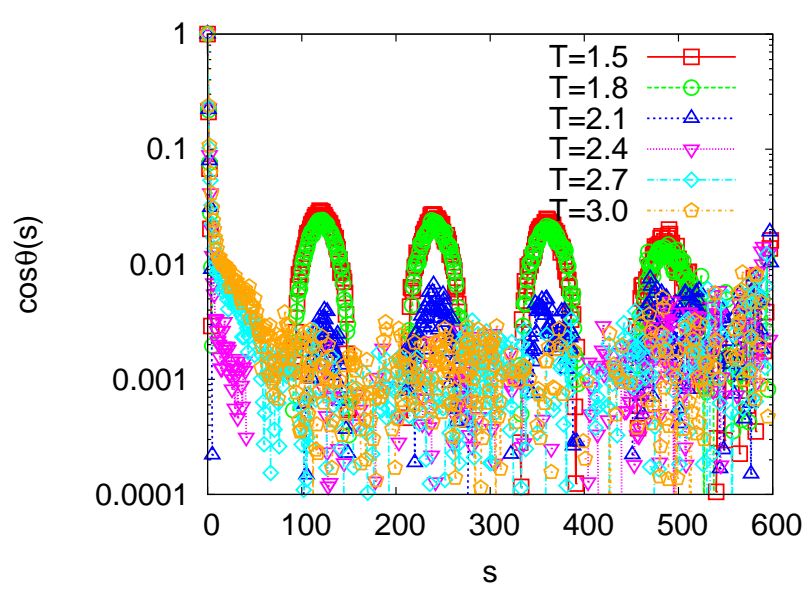

(a)

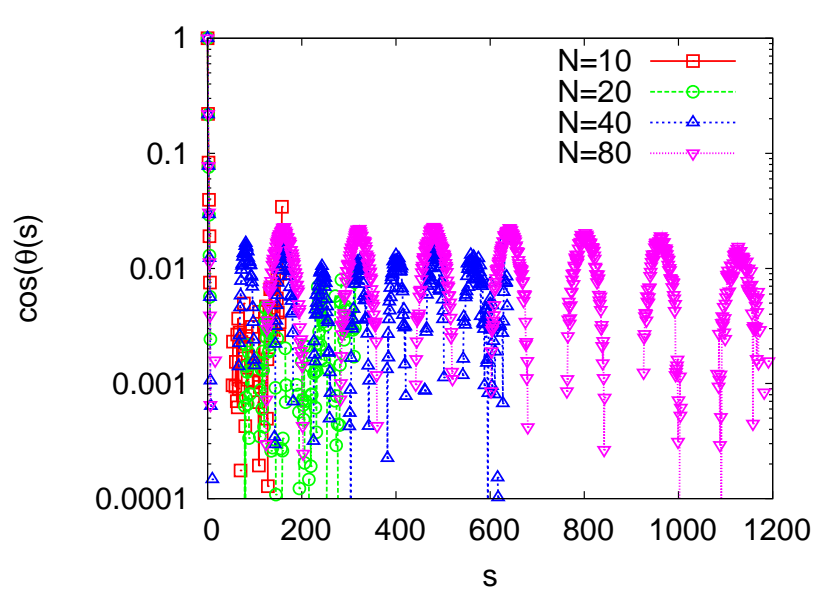

(b)

FIG. 7: (Color online) Bond orientational correlation for the case $n=10, N=60$ (a) corresponding to a full phase separated system at temperature below $T=2.4$

(here $T=1.5$ ), i.e. all blocks of type A join in one cluster while the blocks of type B form another cluster.

At temperatures $T>2.4$ the chain obtains coil-like formations. The dependence on the block length $N$ for chains with $n=16$ is shown in part (b). For block length $N>20$ the phase separation between blocks A and $\mathrm{B}$ takes place. These data refer to the temperature

$$
T=1.8 \text {. }
$$

plateau-like regime. We can even observe a slight increase as temperature decreases and the incompatibility of $\mathrm{A}$ and B monomers increases. As $n$ increases ( $N$ decreases) this plateau-like dependence appears correspondingly at lower temperature $T$. Knowing already the phase behavior of these systems [13 15], these temperatures correspond to those that the multiblock copolymer chain separates in two clusters with a single A-B interface forming between them, where each cluster contains only beads of 
one type with a permanent interface A-B separating the two clusters. The transition of multiple A-B interfaces to a single A-B interface becomes also noticeable in the behavior of asphericity, for high $n$, i.e. $n>20$, where this plateau now has disappeared. Therefore, the phase behavior of the multiblock chains is nicely reflected to a quantity that describes the overall formation of the chain, without taking into account the measurement of any microscopic quantities which relate to the incompatibility between $\mathrm{A}$ and $\mathrm{B}$ beads.

For the case $n=2$ this transition to the phase separated clusters can not be concluded from the results of Fig. 3. because in this case the chain always has only these two clusters of $\mathrm{A}$ and $\mathrm{B}$ beads either in a coil like formation at higher temperatures or a globule-like formation at lower temperatures. In this case of $n=2$ we rather see a small decrease in the quantity $b / S$, as the chain obtains a globular formation. Also, the values of $b / S$ for $n=2$ are higher than the other cases indicating the stronger presence of A-B interface. In this case a smaller number of unfavorable contacts A-B exist (Fig.4b of Ref. [13]). For all $n$ shown in Fig. 3 a more spherical formation is obtained at lower temperatures. In particular this spherical symmetry is strongly present for the multiblock chains of small $N$, where also we do not have the appearance of this large A-B interface and therefore the curves vary smoothly with the temperature $T$. The above description is valid for all cases where full phase separation occurs without restrictions on the length $N$ or number of blocks $n$ and reflects totally the full phase separation behavior of multiblock copolymers.

Now we turn our focus on acylindricity and asphericity for the blocks and the whole multiblock chains on their dependence with the block length $N$ and the number of blocks $n$, so without the fact that results correspond to chains of the same total length $n N$. Figure 4 a shows such results for the average acylindricity of individual blocks and its dependence on the block length $N$ for a multiblock chain containing $n=8$ blocks. It has been shown that for this number of blocks phase separation in two clusters A and B is taking place for high enough block lengths $(N>20)$ following our simulation protocol. However, the plot of this quantity for individual blocks does not reflect the different phase separation behavior for such systems. At temperatures close to the $\Theta$, i.e. $T=3.0$, an almost straight line is seen suggesting that chain blocks appear with the same overall formation irrespective of their block length $N$. At lower temperatures down to $T=1.5$ deviations are seen from the above behavior and the chains with higher $N$ show a higher dependence on the temperature $T$. Larger blocks tend to obtain a more cylindrical-like shape as the temperature decreases at $T=1.5$, something that is seen for all cases.

However, it becomes clear that even for small $N$ the increase of the number of blocks $n$ favors a more cylindrical shape of individual blocks on average. In the case that the chain length $N$ is high enough that full phase separation can take place $(N=80$, Fig. 4 $\mathrm{b})$, i.e., one

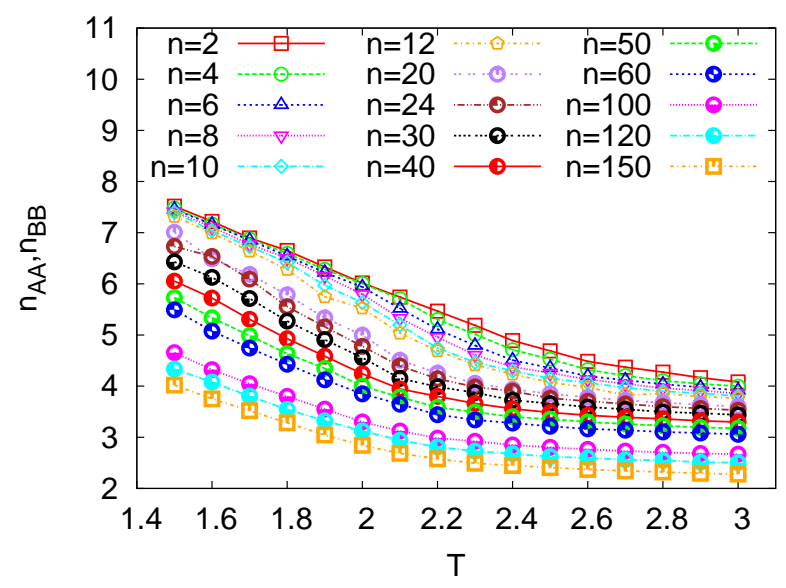

(a)

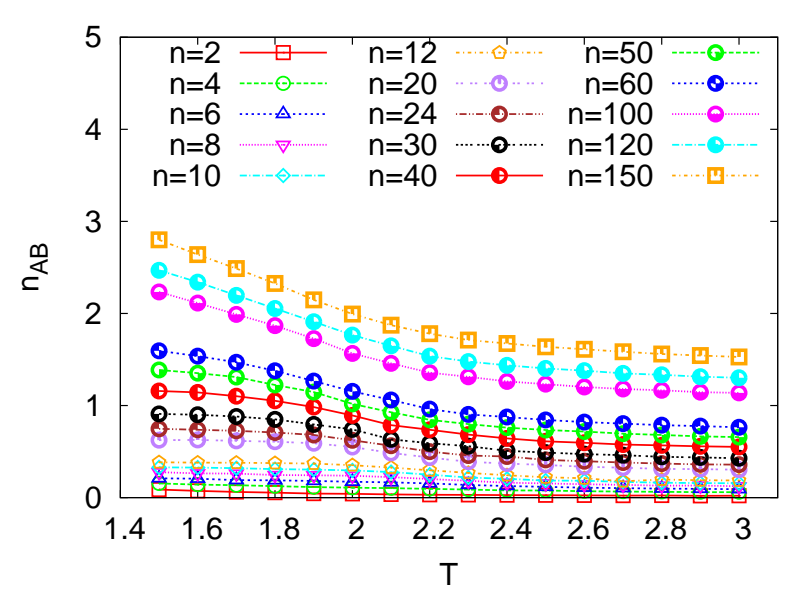

(b)

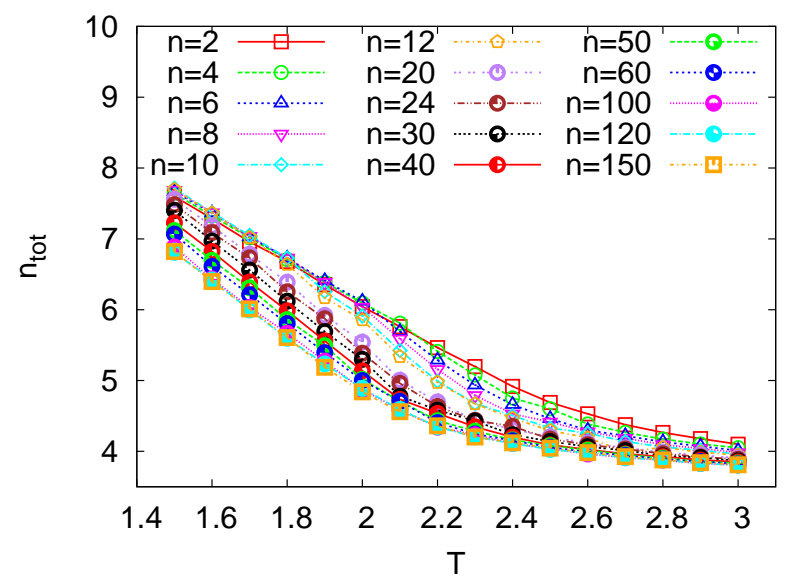

(c)

FIG. 8: (Color online) The dependence of the number of contacts on temperature for different $n$ for chains of total length $n N=600$. Contacts AA (a), AB (b), and the total number of contacts irrespective of whether they are of case A or B (c) are shown. The fraction of A and $\mathrm{B}$ monomers is the same for cases, as the total length of the multiblock chain is kept constant and the most symmetrical case is considered, i.e. an even number of blocks of the same length alternate along the chain. Block lengths fulfilling this condition for $n N=600$ are considered. 
cluster contains the beads of all blocks of type A and another cluster beads of all blocks of type B, variations due to temperature can become now more apparent. For $N=80$ we observe the same tendency that is seen for $N=5$, but the chains are long enough to observe the differences attributed to different temperatures. This can also be concluded by examining the $n$ dependence of multiblock chains with various block lengths $N$. The graphs of Fig. (4 show that the shape of individual blocks is affected by the number and block of lengths, but phenomena of phase separation can not be identified on an individual block-based analysis as a function of block length $N$ or number of blocks $n$.

Plots showing the overall asphericity of multiblock chains as a function of $N$ or $n$ (Fig. 5) neither do reveal any entry to the phase separation regime of multiblock chains and the behavior is similar to what is seen for the average properties of the individual blocks. Asphericity shows an independence on $n$ and $N$ for temperatures close to $\Theta$ (i.e. $T=3.0$ ), while $b / S$ decays with $N$ or $n$ obtaining a more globular formation with decreasing temperature and increasing $n$ or $N$, indicating the entry to the regime where the multiblock chain collapses in a poor solvent. Showing the corresponding plots of Fig. 4 for the asphericity of the blocks (Fig. 6), we draw the same conclusions. However, the asphericity of the blocks shows a more pronounced relation to the entry of the globule regime. Higher block lengths exhibit a higher dependence on the change of temperature and decreasing the temperature from $T=2.4$ to $T=1.5$ (Fig. 6a) the slope of the curve does not change, implying that regarding asphericity of individual blocks there is a stronger dependence on $N$, rather than $n$ (Figs. 6b).

As in the case of Fig. 3 where we have illustrated the asphericity of a multiblock chain as a function of temperature, the phase behavior of multiblock copolymer chains can be perfectly reflected to other properties, as for example the orientational correlation function of Eq. (5). Then Fig. 7 shows examples of this quantity for different cases. For a system not exhibiting full phase separation this orientational correlation (not shown here) for high values of distance $s$ (defined in number of beads along the chain) is usually neglected, since in this range of $s$ we rather observe random scattered points indicating a loss of correlation. However, as we will see this range of $s$ could provide very important information in the case of multiblock copolymer chains, when a single interface between blocks A and B forms for various $n$ and $N$ at low temperatures $T$. For $s$ close to zero (Fig. 7) one is not able to fit any exponents, rather there occurs a very fast decay, which is more pronounced at lower temperatures. It is remarkable that this initial decay is independent of the varied parameters; an obvious interpretation is that in the scale of a few subsequent bonds the chain maintains a high local flexibility.

We focus now on our aim which is to relate the phase behavior to the bond orientational correlations, which is very well shown in Figs. 7 $\mathrm{a}$ and b. Figure $7 \mathrm{l}$ shows

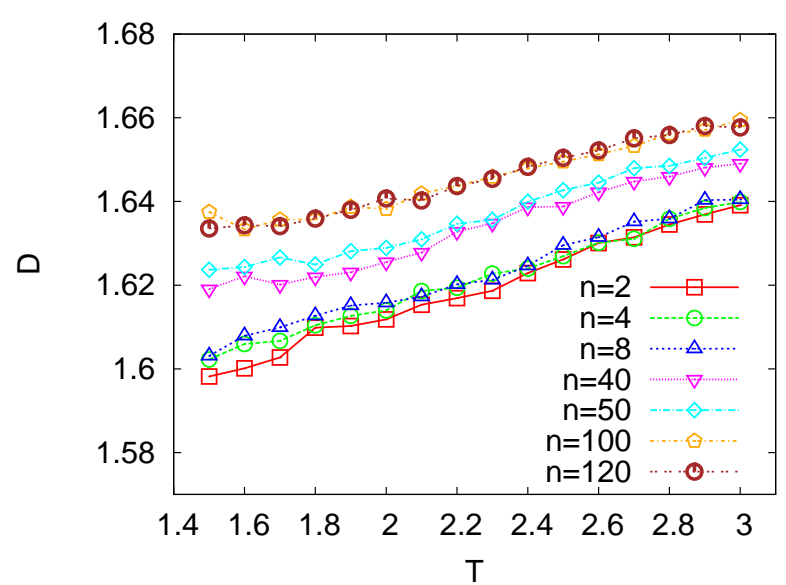

(a)

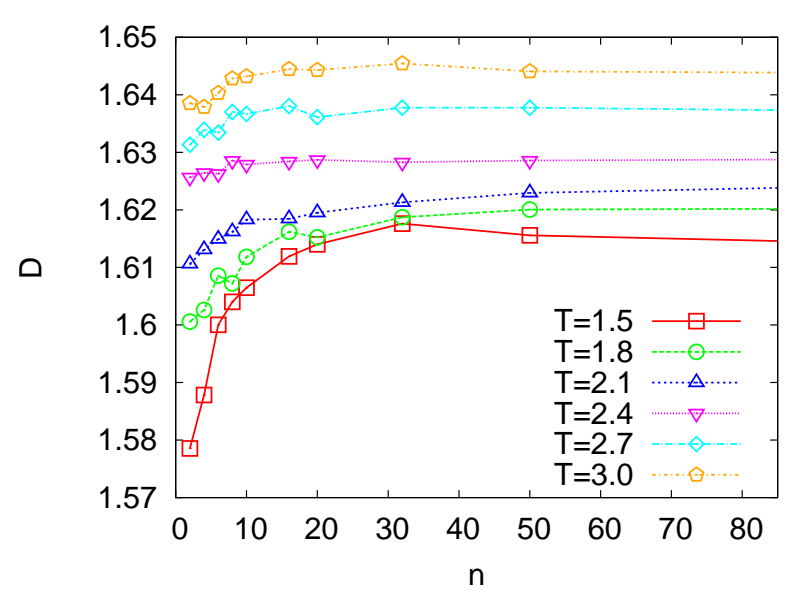

(b)

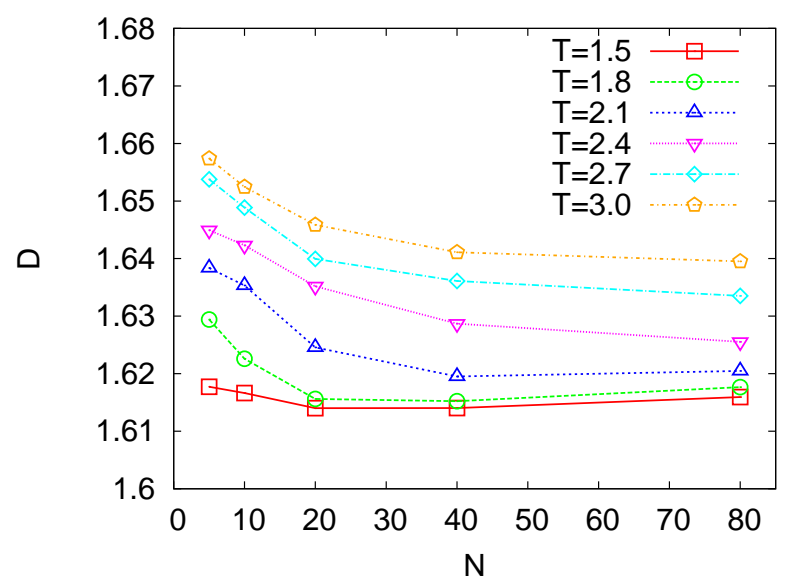

(c)

FIG. 9: (Color online) The dihedral angle as a function of temperature for chains of total length $n N=600$ (a). The dependence of the same property for chains with $N=40$ with the number of blocks $n$ (b), and for chains with $n=20$ with the block length $N$ (c). $D$ is measured in radians. 


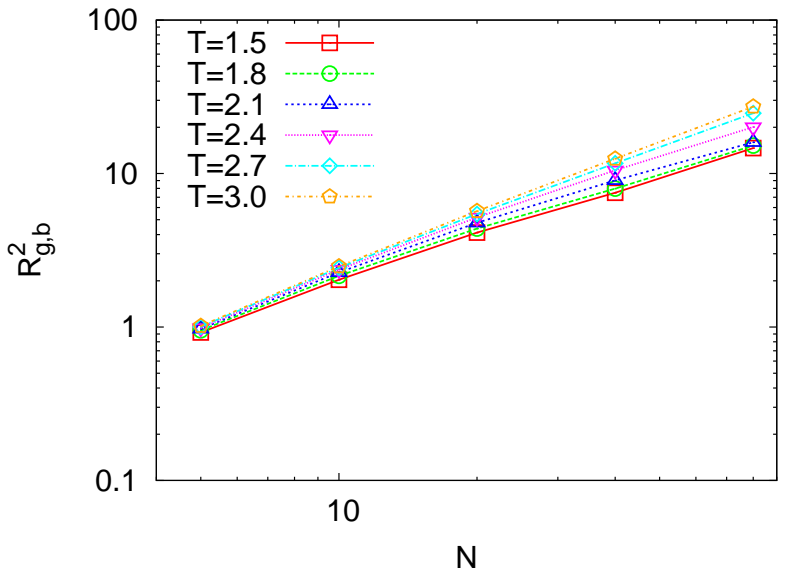

(a)

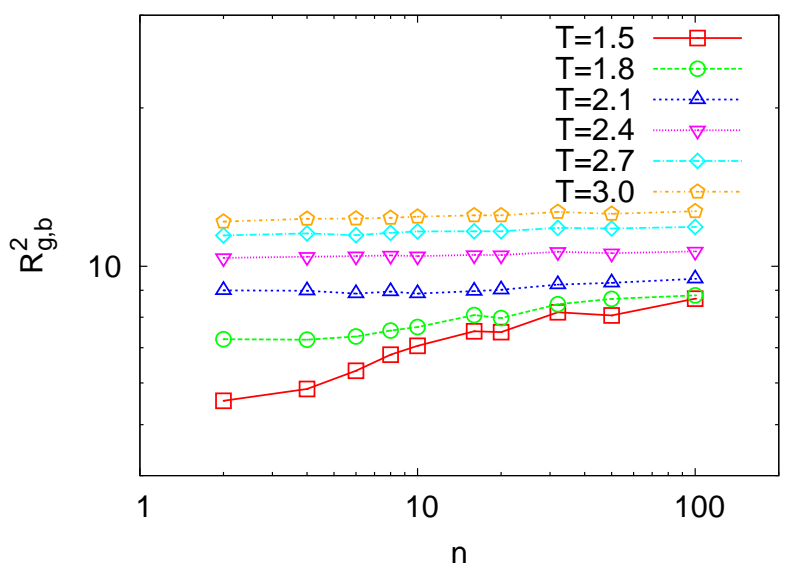

(b)

FIG. 10: (Color online) Two representative cases for the mean square gyration radius of the blocks for chains with $n=20$ (a), and for chains with $N=40$ (b) for different temperatures as indicated.

results for a multiblock copolymer chain with $N=60$. At temperatures $T \leq 2.1$ and $s>60$ we observe peaks which are repeated periodically, as $s$ increases. We can clearly observe four different peaks, which correspond to the $(n-2) / 2$ consecutive A-B blocks respectively. The centers of these peaks correspond exactly to an integer multiple of the total length of an A and B block, which are joint together one after the other. These peaks appear for the range of temperatures that a complete phase separation of A and B blocks takes place (a single interface between A and B blocks is formed). Then the values at small $s(s<2 N)$ correspond to correlations of bonds within the length of a block A and B. Again, the orientational correlation function is able to provide very good information for intermediate and high $s$ for the phase behavior of multiblock copolymer chains under poor solvent conditions. Figure $7 \mathrm{~b}$ shows results for the case $n=16$ at $T=1.8$ for chains of different block length $N$. At this temperature, systems that exhibit a full phase separation between blocks $\mathrm{A}$ and $\mathrm{B}$ show symmetrical peaks at positions $2 l N$, where $l$ takes values $2,3, \ldots,(n-2) / 2$.

The number of contacts of beads does not also reveal directly any correspondence to the phase behavior of multiblock copolymers (Fig. 8), although these numbers are a direct result of the microscopic interactions between monomers. The number of contacts A-A (which is equal to the number of contacts B-B due to the symmetry of our model) decreases monotonically with increasing temperature as beads come apart, but with a lower rate for temperatures $T>2.4$, as we can see from Fig. 8a. We have found [13] that this is the temperature that the chain enters the collapsed state, i.e. effects due to the poor solvent are becoming very important until the chain obtains a globular formation at even lower temperatures. For different number of blocks $n$, this change in the slope sets in at different temperatures. Of course, for symmetric multiblock copolymer chains of the same total length $n N=600$ and increasing $n$ it is expected that the number of contacts A-A will become lower. Increasing the number of blocks and joining them one after the other, the number of contacts A-B increases. This is better shown in Fig. 8b. Counting the number of contacts between beads irrespective of their type (Fig. 8f), we can see that the increasing number of blocks $n$ for chains of the same total length $n N$ results in smaller amount of contacts between beads. This is obviously attributed to unfavorable contacts $\mathrm{A}-\mathrm{B}$; beads $\mathrm{A}$ and $\mathrm{B}$ prefer to be apart, reducing in this way the number of contacts $n_{t o t}$ for chains composed of the same amount of beads $\mathrm{A}$ and B. Again, such plots do not provide a clear information on the regime that this phase separation between blocks in a multiblock copolymer chain takes place. We rather see a change in the slope of the curves upon entering the collapsed state, and the results of Fig. 8 suggest that any transitions take place gradually.

As the bond orientational correlation has shown a direct correlation on the phase behavior of a multiblock chain, we have also looked at other properties as a function of $n, N$, and $T$, as it is for example the dihedral angle $D$ which is formed by four consecutive monomers and taking the average of this quantity along the chain for all $n N-3$ tetrads disregarding in this way effects attributed to different sequences along the chain. As the orientational parameters for small correlation distances $s$ in terms of monomer count (e.g. $s<4$ ) has shown, the chain keeps its local flexibility irrespective of the interactions A,B, or even the chain architecture 37]. Therefore, small differences in $D$ are expected with variation of the parameters $n, N$, and $T$ in the case of multiblock copolymers, as it also appears in Fig. 9. Then, Fig. 9a illustrates the behavior of $D$ with temperature $T$. Increase of the temperature induces a slight increase in $D$ for all chains with different number of blocks, that is a tiny local stretching of the chains. The increase in the 


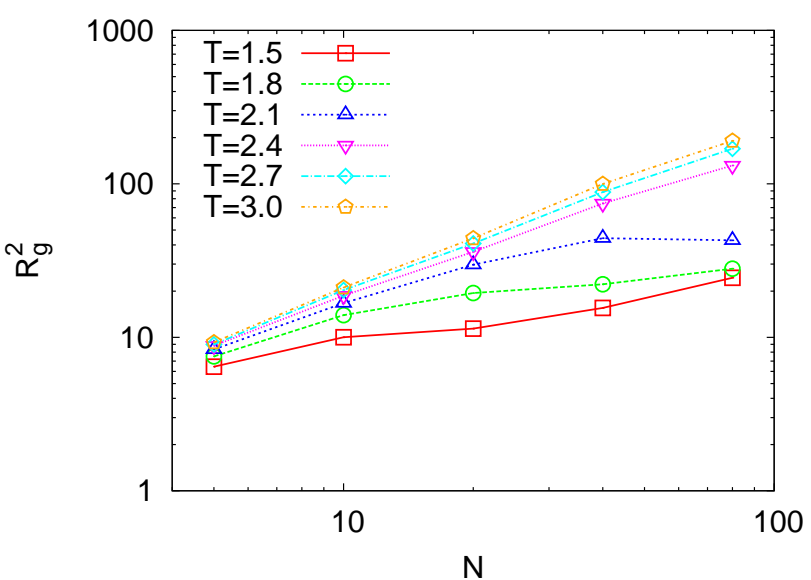

(a)

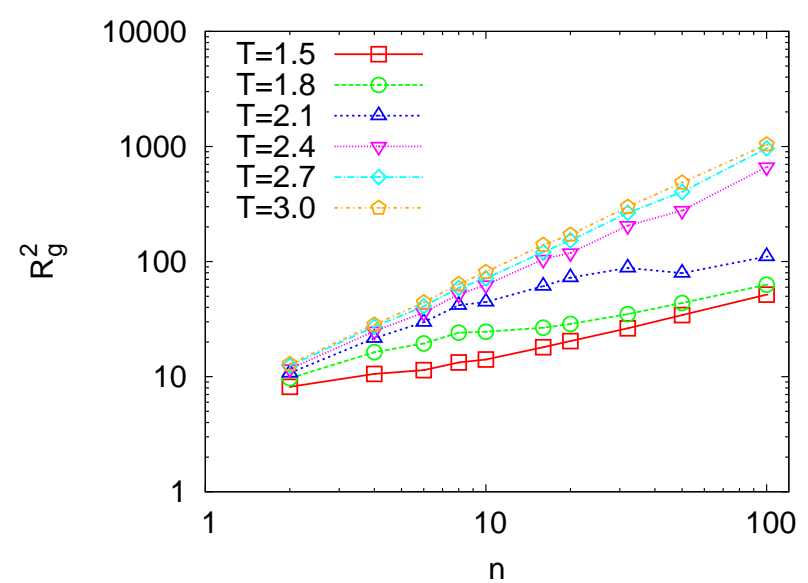

(b)

FIG. 11: (Color online) Two representative cases for the mean square gyration radius for chains of $n=10$ as a function of the block length $N$ (a), and for chains of $N=20$ as a function of the number of blocks $n$ (b).

number of blocks $n$ shows that $D$ also increases, but this change is rather small due to reasons that have already been discussed. In this case all chains have the same total length $n N=600$. If we choose a rather intermediate block length (as for example in Fig. 9] , $N=40$ ), we can see the dependence on $n$. However, in this case and for $n>20, D$ has reached a plateau. For small number of $n$ there is a sharper increase of $D$ with $n$, which is also more pronounced at lower temperatures and in the range $n<10$. But, this is not the whole story, $D$ depends also on the block length $N$ (see Fig. 9f). Here we show an example for $n=20$, which corresponds to a point belonging to the plateau regime of part (c) of Fig. 9. $D$ provides interesting information on the local behavior of multiblock copolymer chains, with small differences arising by variation of the parameters and no effects related to the phase behavior of the multiblock copolymers can be found, as expected, since $D$ is a particularly local property of the chain.

Figure 10] shows the dependence on $N$ for a choice of intermediate number of blocks $n=20$ for different temperatures. Differences due to temperature are expected to increase as the block length $N$ increases. We see that small differences result from the variation of $N$ for different temperatures. Similar behavior we see for other values of $n$. Interestingly the size of individual blocks also depends on the number of blocks $n$ (Fig. 10b) at lower temperatures, but at higher temperatures the gyration radius of the blocks does not change with $n$. Again, these are results for an intermediate value of block length $N=40$, where a full phase separation between A and $\mathrm{B}$ blocks can take place at low temperatures. One could argue that the number of blocks does not play any role when the chain has a coil formation, while in the collapsed state a $n$-dependence occurs at low number of blocks $n$. However, a connection of phase separation or a partial phase separation between blocks is not seen for this property of individual blocks by examining all relevant cases and possible variations of parameters. Nevertheless, it shows us that the dependence on $n$ of the individual size of the blocks seen under poor solvent conditions, is smeared out at high number of blocks $n$. Similar effects are seen for other choices of $N$. Unfortunately, increasing the number of blocks $n$ for the interesting range of $N$ and apply further exploration of this phenomena is rather limited in the present simulations.

Assuming a rather small number of blocks $n$, and knowing that full phase separation is favored by a small number of blocks, we plot the squared average gyration radius of the whole multiblock chain (Fig. 11a). At temperatures close to $\Theta$ one can see a straight line (mind the double logarithmic scale), as expected. Deviations from this behavior are seen for lower temperatures. The dependence on the number of blocks $n$ is shown in Fig. 11] In both figures we can rather see the same behavior for variation of $n$ and $N$, showing that the overall dimensions of the multiblock copolymer chain rather do not depend very much on the way that the blocks are distributed along the chains. Of course, increasing the number of blocks favors a more elongated chain at lower temperatures, due to the higher number of unfavorable interactions between A and B monomers. Similar conclusions are drawn by examining all combinations of $n, N$, and $T$.

\section{SUMMARY}

In this manuscript we have presented results for a single multiblock chain and its individual blocks varying 
parameters such as the block length $N$, the number of blocks $n$, and the temperature $T$. The phase behavior of multiblock copolymer chains in a dilute solution has been discussed in detail elsewhere [14, 15], as well as the static properties of the clusters composed of blocks A or B [13], which are formed due to the microphase separation of monomers A and B. In this manuscript we have tried to make a connection between the phase behavior of these macromolecules and the static properties of the chain and of the individual blocks on average. We have shown that properties related to the chain as a whole can reveal significant information on the phase behavior of multiblock copolymers, while study of the individual blocks does not provide such information. The presence of a single A-B interface between blocks of different type play the major role in the behavior of multiblock chains. The effect of the presence of this interface is reflected to properties such as the bond orientation correlation function and the asphericity of the whole multiblock chains. In particular, we have shown that correlations for intermediate and high distances $s$ (measured in number of monomers) along the chains for the orientation correlation function which, is generally not interesting for a homopolymer chain, can show very interesting effects due to the presence of a well defined A-B interface in the case of multiblock copolymers. Furthermore, it is remarkable that the study of properties of the individual blocks can reveal dependence on parameters such as the number of blocks $n$ as the chain gets into the collapsed state. This dependence is lost for higher number of blocks. Phase separation that is due to the microscopic interactions between monomers and the incompatibility of $\mathrm{A}$ and $\mathrm{B}$ monomers can be mainly measured with properties related to the chain as a whole, or on the basis of a cluster analysis for multiblock copolymer chains, as was done previously 13 15].

The results presented in this manuscript, in com- bination with recent results in the phase behavior of multiblock copolymer chains, give a detailed description for these systems, at least for the most symmetrical case, where an even number of blocks of A and B type monomers alternate along a linear multiblock chain with $N_{A}=N_{B}$ being the lengths of blocks A and B respectively. It is interesting to see that the change of composition of such symmetrical chains can lead to different scenarios of phase separation. Introducing any kind of asymmetry in our systems or the consideration of an additional type of monomers in the structure of multiblock chains could result in different and interesting behavior for these systems. The use of a standard generic coarsegrained model to describe our chains could act as a reference system for the study of these complicated systems. Moreover, the behavior of multiblock copolymers can be parallelized to that of various biological macromolecules which are formed by periodically repeated chemical units ("monomers") along their chain. A detailed discussion on the properties of multiblock copolymer chains using a simple model for a variety of parameters $n, N$, and $T$ is useful to understand the behavior of these chains, before any other effects, due to, e.g. charges, more complicated interactions, architecture, etc., come into play. We envisage that the present contribution could also stimulate further work on these topics.

\section{Acknowledgments}

P.E.T. is grateful for financial support by the Austrian Science Foundation within the SFB ViCoM (grant F41). He also acknowledges financial support through a Max Planck fellowship awarded by the Max Planck Institute for Polymer Research at an initial stage of this work.
[1] G. S. Bates and G. H. Fredrickson, Annu. Rev. Phys. Chem. 41, 525 (1990).

[2] P. D. Topham, A. J. Parnell, and R. C. Hiorns J. Polym. Sci. Part B: Polym. Phys. doi: 10.1002/polb.22302 (2011).

[3] M. W. Matsen and M. Schick Macromolecules 27, 7154 (1994).

[4] M. Matsen and M. Schick Phys. Rev. Lett. 72, 2660 (1994).

[5] M. W. Matsen and M. Schick Macromolecules 27, 6761 (1994).

[6] M. W. Matsen J. Chem. Phys. 102, 3884 (1995).

[7] H. Fried and K. Binder J. Chem. Phys. 94, 8349 (1991).

[8] K. Lewandowski and M. Banaszak Phys. Rev. E 84, 011806 (2011).

[9] K. Lewandowski, P. Knychała, and M. Banaszak Phys. stat. sol. (b) 245, 2524 (2008).

[10] K. Lewandowski and M. Banaszak J. Non-Crystal. Sol. 355, 1289 (2009).
[11] O. F. Olaj, B. Neubauer, and G. Zifferer Macromol. Theory Simul. 7, 171 (1998).

[12] O. F. Olaj, B. Neubauer, and G. Zifferer Macromol. Theory Simul. 7, 181 (1998).

[13] N. G. Fytas and P. E. Theodorakis J. Phys.: Condens. Matter. 23, 235106 (2011).

[14] P. E. Theodorakis and N. G. Fytas EPL 93, 43001 (2011).

[15] P. E. Theodorakis and N. G. Fytas Soft Matter 7, 1038 (2011).

[16] K. Yue and K. A. Dill Proc. Natl. Acad. Sci. USA 92, 146 (1995).

[17] T. E. Creighton Protein Folding (Freeman, New York) 1994.

[18] S. Wołoszczuk, M. Banaszak, P. Knychała, K. Lewandowski, and M. Radosz J. Non-Crystal. Sol. 354, 4138 (2008).

[19] T. T. Pham, B. Dünweg, and J. R. Prakash Macromolecules 43, 10084 (2010). 
[20] S. K. Das, J. Horbach, and K. Binder J. Chem. Phys. 119, 1547 (2003).

[21] P. E. Theodorakis, W. Paul, and K. Binder EPL 88, 63002 (2009).

[22] P. E. Theodorakis, W. Paul, and K. Binder J. Chem. Phys. 133, 104901 (2010).

[23] G. S. Grest and K. Kremer Phys. Rev. A 33, 3628 (1986).

[24] G. S. Grest and M. Murat monte Carlo and Molecular Dynamics Simulations in Polymer Science ed K. Binder (Freeman, New York) 1995.

[25] G. S. Grest Macromolecules 27, 3493 (1994).

[26] G. S. Grest and M. Murat Macromolecules 26, 3108 (1993).

[27] P. E. Theodorakis, W. Paul, and K. Binder Macromolecules 43, 5137 (2010).

[28] P. E. Theodorakis, W. Paul, and K. Binder Eur. Phys. J. E 34, 52 (2011).

[29] I. Erukhimovich, P. E. Theodorakis, W. Paul, and K. Binder J. Chem. Phys. 134, 054906 (2011).

[30] W. Graessley, R. C. Hayward, and G. S. Grest Macromolecules 32, 3510 (1999).

[31] W. F. van Gunsteren and H. J. C Berendsen Mol. Simul. 1, 173 (1988).
[32] http://www.gromacs.org

[33] P. J. Flory Statistical Mechanics of Chain Molecules (Intersience, New York) 1969.

[34] A. Yu. Grosberg and A. R. Khokhlov Statistical Physics of Macromolecules (AIP Press, New York) 1994.

[35] M. Rubinstein and R. H. Colby Polymer Physics (Oxford Univ. Press, Oxford) 2003.

[36] H.-P. Hsu, W. Paul, and K. Binder Macromolecules 43, 3094 (2010).

[37] P. E. Theodorakis, H.-P. Hsu, W. Paul, and K. Binder J. Chem. Phys. 135164903 (2011).

[38] D. Shirvanyants, S. Panyukov, Q. Liao, and M. Rubinstein Macromolecules 41, 1475 (2008).

[39] P. Beckrich, A. Johner, A. N. Semenov, S. P. Obukhov, H. Benoit, and J. P. Wittmer Macromolecules 40, 3805 (2007).

[40] D. N. Theodorou and U. W. Suter Macromolecules 18, 1206 (1985).

[41] S. Hadizadeh, A. Linhananta, and S. S. Plotkin Macromolecules , doi: 10.1021/ma200454e (2011).

[42] F. H. Stillinger J. Chem. Phys. 38, 1486 (1963). 